\title{
VulnerabiLidad Y SU USO EN LA POLÍtica SOCIAL DEL ESTADO de YUCATÁN. La Dirección de Atención a la Infancia y la Familia
}

\author{
Vulnerability and its Use in Social Policy of the State of Yucatán: \\ The Bureau of Assistance to Children and Families
}

\author{
Amada Rubio-Herrera \\ Fátima Flores-Palacios
}

Resumen: Este trabajo tiene el objetivo de analizar cómo se usa el concepto de vulnerabilidad en el discurso oficial de la Dirección de Atención a la Infancia y la Familia del Sistema Nacional para el Desarrollo Integral de la Familia en el estado de Yucatán, México. Siguiendo el método de la etnografía institucional, se entrevistó a funcionarios de la institución y se revisaron documentos generados desde ella. Se halló que el discurso oficial difiere de las implicaciones reales del concepto, principalmente en relación con la multidimensionalidad y la estructura que presentan las experiencias de las personas vulnerables. Se reflexiona sobre la importancia de los contextos situados para comprender las historias de riesgo y proponer estrategias de acción.

Palabras clave: vulnerabilidad, desarrollo, políticas públicas, género.

Abstract: The goal of this paper is to analyze how the Bureau of Assistance to Children and Families of the State of Yucatan, Mexico, uses the concept of vulnerability in its official discourse. Using institutional ethnography, we interviewed civil servants from the Bureau and reviewed documents regarding their activities. We demonstrate that official discourse differs from the real implications of its use, mainly in terms of the multidimensional character and structure that arise from the experiences of vulnerable people. In this article, we reflect on the importance of situated contexts in order to gain greater understanding of the histories of risk and to propose strategies of action.

Keywords: vulnerability, development, institutions, public policies, gender.

\footnotetext{
Amada Rubio Herrera. Doctora en Antropología por la Universidad Nacional Autónoma de México. Becaria posdoctoral del Programa Becas Postdoctorales en el Centro Peninsular en Humanidades y en Ciencias Sociales (CEPHCIS) de la Universidad Nacional Autónoma de México. Temas de especialización: género, desarrollo, etnografía institucional, vulnerabilidad. Correo electrónico: alexarhm@yahoo.com.

Fátima Flores Palacios. Doctora en Psicología por la Universidad Autónoma de Madrid, España. Investigadora en el Centro Peninsular en Humanidades y en Ciencias Sociales (CEPHCIS) de la Universidad
}

Nacional Autónoma de México. Temas de especialización: representaciones sociales, género, salud, vulnerabilidad, salud mental y VIH-SIDA. Correo electrónico: fatimafpalacios@gmail.com.

Enviado a dictamen: 2 de octubre de 2017

Aprobación: 16 de abril de 2018

Revisiones: 1 


\section{Introducción}

$\amalg$ concepto "vulnerabilidad" se ha empleado en diferentes contextos de investigación desde las ciencias sociales. Un primer acercamiento permite identificar el término como adjetivador para una situación anómala, o bien como indicativo de una serie de características que definen a ciertos grupos sociales, por ejemplo, a las mujeres pobres, indígenas, discapacitadas y desempleadas. Sin embargo, el concepto es más que la suma o el recuento de adjetivos; su carga semántica implica una estructura socioeconómica explicativa de las situaciones o formas específicas en las cuales se configura la propia vulnerabilidad.

El uso oficial del término en el discurso que rige a programas y proyectos del estado de Yucatán, México, dista de reflejar la estructura que subyace en las historias de los grupos sociales históricamente construidos en la indefensión, como las mujeres indígenas. Esto tiene como resultado que las acciones emprendidas para abordar temáticas como la salud, la alimentación o el empleo se concentren en necesidades inmediatas, y no estratégicas, lo cual garantiza una incidencia tenue en las condiciones de vida de esa población, ancladas en inequidades que se apuntalan en el no reconocimiento del otro como un igual, en este caso de las mujeres indígenas, pobres y vulneradas.

El objetivo de este trabajo es analizar cómo se utiliza el concepto "vulnerabilidad" en el discurso oficial desde una institución dedicada al desarrollo social en el estado de Yucatán, México: la Dirección de Atención a la Infancia y la Familia (DAIF) del Sistema Nacional para el Desarrollo Integral de la Familia (DIF). Se retoma la etnografía institucional, entendida como una propuesta metodológica cualitativa surgida en el seno de la sociología a través de la obra de Dorothy E. Smith, que se caracteriza por mostrar cómo el orden institucional da pautas para experiencias concretas. Se trata de un método que devela el funcionamiento de una institución en su lógica interna, desde su entramado de relaciones de poder que configuran las experiencias personales de quienes se vinculan con ella. A partir de este método pueden aprehenderse los procesos de institucionalización y las formas en las cuales experiencias concretas suelen tornarse en versiones y datos asimilables para la institución (Smith, 2005). En México, esta perspectiva ha sido utilizada en investigaciones antropológicas para analizar programas gubernamentales específicos (Vizcarra, 2002; Hevia, 2009; Vizcarra y Farfán, 2012).

En el caso concreto estudiado en este texto, se analizó el discurso oficial que sustenta la DAIF para comprender cómo la institución concibe y opera, en el discurso y en la práctica, sus programas, que posteriormente son aplicados en pro de los sujetos definidos como vulnerables, quienes finalmente los experimentan. Este ejercicio se realizó en dos sentidos: en primer lugar se revisaron los contenidos de documentos institucionales disponibles para develar los discursos oficiales que dieron origen y sustentan el concepto de vulnerabilidad desde la política estatal; también interesó aprehender el empleo de dicho concepto desde la lógica institucional, de tal manera que de él derivan propuestas concretas de intervención como las de la DAIF. Los documentos analizados fueron el Plan estatal de desarrollo 2012-2018 Yucatán (Gobierno del Estado de Yucatán, 2013), el Catálogo de programas de bienes y servicios Yucatán (Gobierno del Estado de Yucatán, 2017), la Ley sobre el Sistema Estatal de Asistencia Social de Yucatán. Decreto núm. 353 (1986) y documentos internos del DIF, principalmente los informes anuales de su presidenta entre los años 2013 y 2016.

En segundo lugar, se realizaron entrevistas semiestructuradas a empleados de la DAIF con base en un muestreo a conveniencia, entendido como la elección de la población de estudio por medios no aleatorios, en el que los criterios de selección se determinaron por la disponibilidad del personal de la institución y su interés por participar en el estudio. Se entrevistó a empleados con diversos cargos, incluyendo a la representante, a personas de mandos medios, a responsables de programas, a trabajadores y trabajadoras sociales y a promotores. También se realizó observación, la cual, junto con las pláticas informales 
con los usuarios, proporcionó datos para conocer con más profundidad cómo opera dicha dirección. Los datos de las entrevistas fueron procesados con base en un análisis de contenido, en el que las categorías temáticas permitieron comprender el discurso y las prácticas que sustenta la DAIF desde sus propios agentes.

\section{La vulnerabilidad adjetivada}

El término "vulnerabilidad" presenta diferentes sentidos. En sus usos más comunes refiere a una situación de pobreza y marginación que padecen ciertos grupos sociales como resultado de condiciones históricas, políticas y económicas (Estrada, 2014). Otro de sus usos se relaciona con los sustantivos y adjetivos que lo acompañan, y que dan como resultado hibridaciones como: vulnerabilidad social, vulnerabilidad económica, vulnerabilidad ambiental, vulnerabilidad jurídica y vulnerabilidad demográfica, por mencionar algunas. Lo que se busca con su adjetivación es especificar y acentuar la variable explicativa de la situación de riesgo (Sáez, Pinazo y Sánchez, 2008).

Una primera definición del concepto refiere a la condición estructural que evoca, cuyas expresiones más concretas pueden adquirir aspectos diferentes según la población y sus características; en todo caso, la vivencia estructural toma matices precisos. Busso ha enfatizado que la vulnerabilidad es ante todo "[...] un proceso multidimensional que confluye en el riesgo o probabilidad del individuo, hogar o comunidad de ser herido, lesionado o dañado ante cambios o permanencias de situaciones externas y/o internas" (Busso, 2001: 8).

Esta estructura o pluridimensionalidad que implica el concepto difícilmente puede aprehenderse en su totalidad por cada objeto, caso o fenómeno estudiado; es decir, analizar la vulnerabilidad desde todas las dimensiones que la componen resultaría una tarea cercana a lo imposible, condenada al fracaso, tal y como lo explican Sáez, Pinazo y Sánchez (2008). Es por ello que a menudo se suele enfatizar la dimensión explicativa desde la cual se tratará.
Pese a que remite a un proceso pluridimensional, la vulnerabilidad se configura en lo individual e integra aspectos subjetivos comprensibles a partir de la historia de vida de los individuos (Flores, 2015). Esa historia concreta retorna al medio social circundante de los actores, y da la pauta para explicar el intercambio dinámico y dialógico entre lo individual y lo social; ambas dimensiones son indisolubles en este vínculo.

La vulnerabilidad social ha sido el compuesto que desde las políticas públicas se ha empleado para abordar problemas específicos. De acuerdo con Villa, se trata de una noción explicativa que trasciende el contenido teórico de términos como pobreza, marginalidad y exclusión social, que brinda: "la posibilidad de entender cómo y por qué diferentes actores sociales están sometidos, de modo dinámico y heterogéneo, a procesos que pueden atentar contra su posibilidad de acceder a mayores niveles de bienestar social" (Villa, 2001: 4). Es decir, desde el reconocimiento de la multidimensionalidad del concepto por remitir a causas, fuentes y expresiones diversas, se reivindica el peso de los escenarios sociales, estructurados, que contribuyeron a la situación de riesgo. En este sentido coincidimos con Stern (2004), quien se refiere a la vulnerabilidad social como la combinación de elementos y situaciones que explican, desde su interacción, la existencia de ciertos fenómenos sociales que no pueden ser interpretados desde una sola causa.

Los componentes explicativos a partir de los cuales se articula la vulnerabilidad social suelen ser, por un lado, la indefensión ante el impacto de una situación externa y, por el otro, el empleo de recursos personales, comunitarios o familiares para manejar la situación (Pizarro, 2001). Esto último ha sido definido como "vulnerabilidades recursivas" por Flores, y refiere a las "prácticas resilientes que apoyan la comprensión del suceso o fenómeno vivido o padecido, con el objetivo de buscar un sentido positivo e intentando posicionar al sujeto en una dimensión proactiva, capaz de transformar su propia realidad" (Flores, 2015: 91). La vulnerabilidad recursiva es un proceso que se construye desde la adversidad, es un mecanismo de sobrevivencia que las personas incorporan frente a una situación 
específica de dolor o pérdida, y puede ser material, física o psicológica.

Las características mencionadas particularizan y colocan el término en un nivel de análisis más complejo que, por ejemplo, el concepto de pobreza. Desde su acepción más general, y volviendo a retomar a Villa (2001), la pobreza alude a una carencia de recursos económicos que restringen la satisfacción de necesidades básicas de una población o familia, como por ejemplo las alimentarias o de servicios. Por tanto, las acciones para su erradicación suelen enfocarse en la resolución de las necesidades específicas más apremiantes. En el caso de México, han sido diversos los programas para erradicar la pobreza y la pobreza extrema, entre los que destacan, en los últimos sexenios, la iniciativa Prospera, antes Oportunidades, Progresa y Solidaridad.

No es coincidencia la puesta en marcha de dichas iniciativas en contra de la pobreza en México; en este sentido, la pobreza ha sido una de las principales categorías de análisis, y los indicadores de los diferentes programas buscan demostrar el avance de las políticas en el mejoramiento de las condiciones de vida de los habitantes. De acuerdo con la Organización de las Naciones Unidas (ONU), México ha mostrado avances considerables en el cumplimiento de los Objetivos de Desarrollo del Milenio (ODM), cuyo fin último se dirigía a la erradicación de la pobreza en contextos en vías de desarrollo; la ONU reportó el cumplimiento de seis de los ocho objetivos en el país, aunque algunas metas e indicadores de éstos no se cumplieron en su totalidad (ONU, 2014: 33). Los documentos oficiales consultados, principalmente de la ONU y del Programa de las Naciones Unidas para el Desarrollo (PNUD), aportan información cualitativa y cuantitativa que muestra avances sustanciales en la lucha contra la pobreza extrema y en la mejoría en temas como salud, educación, igualdad de género y medioambiente.

Sin embargo, al analizar casos concretos en contextos situados, entendidos como los espacios comunitarios en los cuales transcurre la vida cotidiana de sus habitantes y en los que se construyen, deconstruyen y reconstruyen representaciones sociales (Flores, 2015), la realidad puede ser diferente, porque ésta muestra que aún hace falta trabajar en la atención de necesidades coyunturales de las poblaciones, aquellas sentidas y vividas en la cotidianidad por sus habitantes, lo cual pondría en entredicho el avance reportado en la agenda desarrollista. Precisamente en este punto radica la importancia del análisis situado: en el reconocimiento de ese saber común, sus potencialidades e inhibidores, su dinamicidad y, sobre todo, las explicaciones que sustentan prácticas y conocimientos cotidianos; por ejemplo, en el caso de interés, cómo se vive subjetivamente la experiencia de vulnerabilidad y cómo se configura y explica desde lo social o contextual. La definición que se utiliza en este texto, en tal sentido, apunta a una dimensión compleja de cierta indefensión ante el contexto situado en que se vive donde predomina, por ejemplo, la falta de oportunidades de empleo y de atención adecuada a la salud, o simplemente el sentimiento de exclusión social, lo que puede generar una comparación desigual en el terreno de los derechos humanos a nivel global.

Recientemente, en la agenda internacional de desarrollo posterior a los ODM se ha declarado que los Objetivos de Desarrollo Sostenible (ODS) constituyen la senda hacia el año 2030, y se remarca el compromiso internacional con el desarrollo inclusivo, en el cual la igualdad de género sea una prioridad. De acuerdo con la página oficial del PNUD, el objetivo 5 de los diecisiete que conforman los ODS busca: "lograr la igualdad entre los géneros y empoderar a todas las mujeres y niñas".

En correspondencia con esa agenda de desarrollo, en el Plan estatal de desarrollo del estado de Yucatán 2012-2018 se señala la importancia de generar una estrategia integral con el propósito de alcanzar el Estado de bienestar, partiendo de:

[...] alternativas de crecimiento sostenibles en el mediano y largo plazos, para lograr un impacto positivo sobre el ingreso y la calidad del empleo, con el enfoque en la igualdad de oportunidades y formación educativa integral, en un territorio con 
servicios sustentables, donde se conserve la paz y tranquilidad que lo caracteriza (Gobierno del Estado de Yucatán, 2013: 38).

En dicho plan se retoman conceptos del discurso internacional del desarrollo como sostenibilidad, igualdad de oportunidades, educación integral y sustentabilidad. Se caracteriza por cinco ejes de acción, el segundo de los cuales, titulado "Yucatán incluyente", muestra los usos que se dan a los conceptos de pobreza y vulnerabilidad en el discurso oficial. El término "pobreza" es empleado para referir a condiciones de vida materiales de determinados sectores del estado, principalmente donde se concentra un alto porcentaje de población mayahablante. Por tanto, el trabajo hacia la erradicación de las carencias materiales conlleva implementar: "acciones para contar con vivienda digna, para disminuir significativamente el fecalismo al aire libre, contar con un programa nutricional y alimentario que permita abatir al máximo los niveles de pobreza extrema, fortalecer las oportunidades de ingreso y la producción de autoconsumo familiar" (Gobierno del Estado de Yucatán, 2013: 78). En el mismo plan se ratifica la urgencia de romper con la correlación entre pobreza y etnicidad, de empoderar al pueblo maya y de promover la educación formal como ruta para el cambio; en este sentido, la pobreza es una condición reversible si se ofrecen los recursos apropiados a quienes los necesitan.

El segundo concepto, "vulnerabilidad", si bien no aparece adjetivado, alude a las condiciones de vida de grupos sociales específicos, principalmente personas con discapacidad, niños, adultos mayores y mujeres. A diferencia de la pobreza, la categoría vulnerabilidad no se restringe o circunscribe a la población maya, sino que es extensible a los habitantes del estado que comparten condiciones de vida particulares y cuyas situaciones de riesgo buscan prevenirse o erradicarse para su inclusión efectiva en todos los ámbitos de la vida social. Sin embargo, se valora que esta particularidad en el tratamiento de la inclusión, desde la vulnerabilidad, equivale a una atención meramente descriptiva de las condiciones de vida de las poblaciones en riesgo, sin un análisis que sustente dicha atención, porque el concepto remite, como se ha mencionado, a una multidimensionalidad que implica consideraciones de mayor amplitud para explicar situaciones de riesgo, y no a una categorización de la población. Más adelante se retomará este punto.

En el eje "Yucatán incluyente" se recalca que una de las labores trascendentales del gobierno estatal es ofrecer calidad en los servicios a la población en riesgo con la finalidad de garantizarles una vida digna:

[...] como parte esencial en las tareas de gobierno, se abordan temas y compromisos concretos en materia accesibilidad urbana, inclusión educativa y fomento laboral para personas con discapacidad, protección social para adultos mayores, apoyo a migrantes y fomento a la no discriminación para la comunidad Lésbica, Gay, Bisexual, Transexual, Travestí, Transgénero, e intersexual (LGBTTTI), que propicien una mayor equidad respecto de las posibilidades de crecimiento humano y profesional, como parte de una comunidad que valore todo lo que los grupos vulnerables pueden contribuir al desarrollo del estado (Gobierno del Estado de Yucatán, 2013: 107, cursivas añadidas).

Otro uso del concepto "vulnerabilidad" que se identificó en el plan de desarrollo está relacionado con el medioambiente, con las características o condiciones de vida materiales que ponen en riesgo al estado y a algunos de sus municipios ante eventos naturales como huracanes u otras situaciones derivadas del cambio climático. Este último uso es frecuente en investigaciones cualitativas, por ejemplo, en el libro Vulnerabilidad y adaptación en Yucatán: un acercamiento desde lo local y con enfoque de género, escrito por Soares y colaboradores (2014). En este texto se hace énfasis en la vulnerabilidad desde las capacidades colectivas para afrontar riesgos provocados por el cambio climático; el nivel de vulnerabilidad está determinado por los capitales sociales, culturales y naturales con los que cuentan los colectivos. Ésta es otra de las características fundamentales del concepto, el grado 
de riesgo en relación con los recursos de que disponen la población o los grupos.

Si se revisa con más detalle la interpretación del concepto "vulnerabilidad" desde el Plan estatal de desarrollo 2012-2018 (Gobierno del Estado de Yucatán, 2013), puede afirmarse que dista de contener las dimensiones estructurales y particulares que implica el término, y aunque en ese documento no se adjetiva explícitamente, sí lo está porque es atribuido a manera de epíteto a grupos alejados de la norma, como las comunidades LGBTTTI o las personas con alguna discapacidad. Esto conduce a que se utilice de manera superflua, como un cliché político que no traspasa el plano discursivo, quedando lejos de aportar a la consecución del objetivo o fin último de la agenda estatal: el Estado de bienestar. En este sentido, "vulnerabilidad" no se diferencia de "pobreza"; ambos conceptos abogan por solucionar necesidades específicas para las personas pobres o vulnerables sin dar cuenta de las situaciones que las originan y explican. Se trata de una utilización fútil de manera que, al obviarse las causas estructurales de las problemáticas, se lacera una vez más a las poblaciones en riesgo.

Para discutir la disonancia existente entre lo que significa el término de vulnerabilidad desde su multidimensionalidad, y su uso en el discurso político del estado y en la misma práctica institucional, se retomará como ejemplo el discurso y la práctica de la Dirección de Atención de la Infancia y la Familia (DAIF), del Sistema Nacional para el Desarrollo Integral de la Familia (DIF), en Yucatán, México.

\section{La DAIF y su quehacer institucional en relación con los grupos vulnerables}

La DAIF es una de las dos subdirecciones generales del DIF estatal, pertenece a la subdirección operativa de la institución y tiene a su cargo diez programas de asistencia social, algunos consolidados y con su reconocimiento correspondiente, como el programa Clubes del Adulto Mayor, y otros de reciente apertura —entre fines de 2016 y principios de 2017- como el programa Promoción del Buen Trato entre las Familias. Otros de sus programas clave son Migración Infantil no Acompañada y Talleres de Sexualidad Responsable.

El objetivo general de la DAIF es la prevención de los riesgos psicosociales que aquejan a la población vulnerable del estado $y$, de acuerdo con el último Catálogo de programas de bienes y servicios Yucatán 2017, en el cual esta institución recibe el nombre de Programa de Atención a la Infancia y la Familia, se considera que esos riesgos son: el abuso, la violencia, la explotación sexual, la migración infantil, las adicciones, los embarazos en adolescentes, el trabajo infantil y la atención al adulto mayor (Gobierno del Estado de Yucatán, 2017). Se parte de considerar que los riesgos psicosociales son condiciones reversibles a partir de la provisión de una serie de bienes y servicios, tales como el otorgamiento de becas y despensas básicas para madres adolescentes o la realización de pláticas, talleres y terapias psicológicas. A través de sus diferentes programas, esta Dirección tiene una amplia cobertura en el estado: en el año 2016 tuvo presencia en 95 de los 106 municipios de la entidad. Su población objetivo está conformada por:

— niñas, niños y adolescentes, adultos, adultos mayo-

res y familias,

— niños en riesgo de trabajo,

- escuelas,

- ayuntamientos.

La DAIF depende del DIF en materia de presupuesto y de recursos humanos. Los cambios de programas, proyectos, responsables y contratación de nuevo personal también dependen del mismo DIF. La responsable de la DAIF durante la realización del estudio (2016-2017), que en el momento de la entrevista hacía dos meses que había asumido el puesto, ${ }^{2}$ comentó que estaba conociendo la Dirección y sus programas, y aseveró que su formación como maestra rural le permitía identificar situaciones de riesgo en contextos situados. Creía que el cambio hacia una sociedad mejor era factible a partir de las actividades sociales que se realizaban en la DAIF, las cuales, agregó, eran de 
orden preventivo. Entre éstas destacó los talleres sobre problemáticas latentes en los contextos situados. En correspondencia, consideraba que la vulnerabilidad es prevenible a partir de las intervenciones que realizaban:

Es exactamente donde tenemos que ver, con la comunidad y con los problemas de la comunidad, y pues es en realidad una labor muy bonita. Le metemos muchas ganas, mucha pasión, aunque a veces salgamos de ahí medio trasquilados, pero la verdad es que hacemos muchas cosas, muchas cosas, como los talleres. ${ }^{3}$

Si bien los orígenes de la DAIF son poco claros, se fundó después de la creación del DIF en el estado a finales del siglo pasado. El DIF se constituyó a partir de un decreto emitido por el gobernador de Yucatán, Víctor Cervera Pacheco, el decreto núm. 353, en el que se define la asistencia social como:

[...] el conjunto de acciones tendientes a modificar y mejorar las circunstancias de carácter social que impidan al individuo su desarrollo integral, así como la protección física, mental y social de personas en estado de necesidad, desprotección o desventaja física o mental, hasta lograr su incorporación a una vida plena y productiva (Gobierno del Estado de Yucatán, 1986: 4).

En concordancia, el DIF se concibió como la institución rectora de la asistencia social en el estado, para articular esfuerzos interinstitucionales destinados a la promoción de acciones de incidencia social. Para este cometido, el decreto 353 le adjudicó veintidós funciones, entre las que sobresalía la capacitación de su personal en materia de servicios de asistencia social como vía para promover y apoyar eficazmente el desarrollo de la infancia y la familia.

En el mismo decreto se discute la conformación de la estructura orgánica del DIF y las funciones de las entidades que la conforman como el Patronato, la Presidencia, la Junta de Gobierno y la Dirección General. Entre sus instancias destaca la Procuraduría de la Defensa del Menor, que desde entonces tiene como función la representación legal de menores en la defensa de sus derechos; la DAIF y otras subdirecciones o entidades no aparecen en ese decreto pues su creación fue posterior a la fundación del DIF en el estado.

Se mencionó previamente que el objetivo de la DAIF es prevenir los riesgos psicosociales en poblaciones catalogadas como vulnerables, llámense niños, mujeres o personas de la tercera edad. Para ello despliega una serie de acciones que buscan impactar sobre esos sectores. Con este compromiso asumido, la presidenta del DIF estatal recalcó en su segundo informe de actividades, de 2015, que durante el ejercicio del año 2014 la DAIF había realizado más de 1123 actividades en los 61 municipios que en ese entonces atendía dicha Dirección, con un total de 58498 personas beneficiadas. Esas actividades consistieron, fundamentalmente, en pláticas y talleres sobre temáticas como embarazo adolescente, adicciones, salud infantil y violencia. También se mencionaba la participación del programa Clubes del Adulto Mayor en los encuentros culturales ofrecidos por la institución. Sin embargo, se considera que esas acciones no apuntalan ni aportan elementos que puedan emplearse para solucionar problemas más profundos e implícitos en la misma condición de vulnerabilidad, por ejemplo, las razones que estructuran el embarazo adolescente o la situación de abandono a la que quedan expuestos los adultos mayores. Esto guarda relación con el mismo proceso de construcción y deconstrucción de representaciones sociales, recuérdese que éstas no son estáticas y se transforman a partir de la experiencia (Flores, 2015). En cuanto al Primer Encuentro Juvenil de Riesgos Psicosociales del año 2014, en el informe referido se hace énfasis en que éste se conformó como un espacio para que los adolescentes expresaran sus inquietudes respecto a temáticas como drogadicción, embarazos y violencia en el noviazgo. Pese a la importancia de dicho encuentro y a que en él se reunieron más de quinientos jóvenes del estado, no se aclara si hubo un seguimiento a esas inquietudes ni cómo se analizaron, en caso de haberse analizado, ni cómo se atendieron 
a posteriori los temas de interés. El hecho de no haber dado seguimiento oportuno a un evento como ese indica que difícilmente se habrá constituido como una experiencia importante en las subjetividades de los jóvenes.

En los datos cuantitativos que se reportan en los informes se observa que la población que más participa en las actividades de la DAIF está conformada por mujeres, desde niñas hasta adultas de la tercera edad. Los empleados de la institución corroboraron esta aseveración, lo que lleva a confirmar la importancia que esta población objetivo tiene para las acciones de desarrollo. Esto no es nuevo y ya en la literatura sobre temas de género se ha demostrado la asidua participación de las mujeres en programas sociales, particularmente en proyectos productivos de desarrollo (Pineda, Vizcarra y Lutz, 2006; Zapata y Suárez, 2007; Rubio y Castillo, 2014).

En el caso del programa Clubes del Adulto Mayor, su responsable, un licenciado en gerontología, comentó que en el padrón de beneficiarios del año 2016 figuraban 1614 personas, la mayoría mujeres. De manera contradictoria, eran ellas quienes, pese a que muchas de ellas continuaban desarrollando largas jornadas de trabajo no reconocidas socialmente, como el cuidado de los nietos, tenían una presencia constante en las actividades desarrolladas por el programa y lo consideraban un espacio fundamental de socialización con sus pares. Ésta es una arista importante de explorar: indagar sobre los efectos de dicho programa en las subjetividades de las participantes ya que, para algunas, la experiencia fue su primer espacio formal de socialización entre iguales fuera de sus hogares.

Villagómez y Sánchez han señalado la poca visibilidad de hombres y mujeres de la tercera edad en las investigaciones que articulan la vulnerabilidad con el género, y en sus estudios analizan situaciones de riesgo padecidas por mujeres rurales de Yucatán y Campeche en México. Centran la atención en las condiciones que exacerban la vulnerabilidad de ese sector, destacando la ausencia de una seguridad económica y social que les garantice una vejez digna, además de las condiciones de pobreza multimensional en sus contextos de vida. También demuestran que, aun en la senectud, las mujeres siguen aportando recursos a sus hogares, manteniéndose activas económicamente y proveyendo de bienes y servicios a la familia (Villagómez y Sánchez, 2013; Villagómez, 2014). Este hallazgo demuestra que, lejos de ser una carga, estas mujeres continúan desarrollando múltiples actividades para la reproducción de sus hogares.

El responsable del programa Clubes del Adulto Mayor aclaró que las personas que más participan son mujeres con condiciones de vida que les permiten tener tiempo para sí mismas; es decir, son aquellas que por lo general reciben mayor apoyo de sus familiares y de ellas no depende la reproducción familiar, pero sí la realización de ciertas actividades como el cuidado de los nietos antes señalado. Retomando las propias historias de vida de las personas entrevistadas como punto de partida, la acotación anterior conduce a pensar en la urgencia de analizar la vulnerabilidad y las diferencias de la aplicación del término considerando elementos como el género, la etnia, la región o la clase entre los adultos mayores.

La vulnerabilidad es definida por los responsables de los programas de la DAIF con base en la población con la que trabajan; el mismo encargado del programa Clubes del Adulto Mayor señaló que el concepto es inherente a las personas de la tercera edad:

Primero que nada, por definición toda persona adulta mayor es considerada vulnerable. El término "vulnerable" es muy amplio, realmente es bastante amplio; aquí lo que nosotros hemos percibido es la falta de apoyo familiar que hay hacia el adulto mayor. Afortunadamente, todavía nuestro estado conserva esa tradición de estar con la familia, que todavía tiene ese apego familiar, pero sí hemos notado casos donde la familia se desentiende completamente del adulto mayor. Ésa es una cuestión primordial y se da más en el aspecto de ciudad que en el aspecto rural. Por ejemplo, en la comunidad todavía vemos que el abuelito vive con la familia, o el abuelito es el que a lo mejor da indicaciones, el que todavía tiene el poder de decisión. ${ }^{4}$ 
Esta manera de entender la vulnerabilidad en los adultos mayores y de argumentar el concepto implica componentes sociales explicativos que indican el riesgo de envejecer en sociedades que se van construyendo sobre dinámicas en las que no se dejan espacios para los adultos mayores. Sin embargo, fue precisamente el representante de dicho programa el que ofreció tales elementos e indicó las limitaciones de este fenómeno que presenta múltiples aristas: la vulnerabilidad entre las personas de la tercera edad.

La mayoría de los entrevistados definió la vulnerabilidad de manera similar a como figura en el siguiente fragmento de entrevista:

[...] en primer lugar, vulnerable es que esté dentro de los programas del DIF; por default, vulnerable es sinónimo del DIF. Vulnerable es de carencia, que tenga alguna carencia, carencia alimentaria, carencia social, carencia económica, isale?, en sí son las que más atendemos... Para nosotros, aquí, en atención a la infancia y la familia, los niños son los más vulnerables. ${ }^{5}$

Esta forma de entender la vulnerabilidad, como se ha mencionado, se centra en la carencia desde su expresión más inmediata sin tomar en cuenta el origen, las dimensiones y las especificidades de cada problemática, ni mucho menos los activos sociales o individuales ante el riesgo, de manera que se considera que estas personas presentan una actitud no proactiva. Esta consideración es una constante en los últimos tres informes (2015-2017) de trabajo de la presidenta del DIF estatal.

En esos informes de trabajo la presidenta del DIF estatal utiliza la palabra "vulnerabilidad" para hacer referencia a una serie de situaciones sociales de carencia, semejantes a las que se indican en el Plan estatal de desarrollo 2012-2018 Yucatán. Enfatiza los logros de la institución en la atención a necesidades apremiantes que aquejan a la población en riesgo, casi siempre citando a grupos de mujeres, niños y discapacitados. Este quehacer del DIF corresponde a su misión como institución según consta en su página oficial:
Brindar servicios de calidad en materia de asistencia social a la población más necesitada y vulnerable del Estado; fortalecer las acciones que contribuyan al desarrollo integral de la familia y propiciar el desarrollo de sus capacidades en un esquema de participación, prevención, equidad e inclusión. ${ }^{6}$

En esos informes también se reitera la advertencia del dato cuantitativo como prueba de las acciones cumplidas por parte de la institución. En otro espacio (Rubio, 2018) se ha señalado que es importante reconocer el dato cuantitativo para validar la eficacia de las acciones institucionales y dimensionar ciertos fenómenos, pero también es fundamental reconocer sus limitaciones al momento de abordar especificidades, como por ejemplo las maneras concretas como se viven las experiencias de vulnerabilidad. El dato numérico opaca esas especificidades, las cuales cobran importancia para analizar la eficacia de las intervenciones gubernamentales. Por ejemplo, el estado de Yucatán, pese a los esfuerzos institucionales, sigue presentando altos índices de pobreza, exclusión y marginación, anclados en condiciones de vida construidas sobre la indefensión y la vulnerabilidad, particularmente en las comunidades rurales del interior del estado. Peor aún si se toma en cuenta que, de manera contradictoria, el estado de Yucatán ocupa un lugar importante en el país en cuanto a crecimiento económico, del 3.7\% en comparación con el 2.5\% de crecimiento nacional. Este dato posiciona a la entidad en un lugar importante en cuanto a inversión y desarrollo económico en el país (INEGI, 2016).

Los últimos datos del Consejo Nacional de Población (CONAPO) indican que en 2010 Yucatán contaba con un grado de marginación alto, y el estado se ubicaba en el lugar número 11 de las entidades del país en relación con este indicador (CONAPO, 2011). En 2015, esta misma institución reportó que más de la mitad de la población del estado, el 57.4\%, vivía en situación de marginación, y el 28\% de ese porcentaje, en marginación extrema (CONAPO, 2016). Estos datos corresponden con la evaluación efectuada por el Consejo Nacional de Evaluación de la Política de 
Desarrollo Social (CONEVAL) para el año 2014, en la cual consta que más del $80 \%$ de la población del estado se encontraba en condiciones de pobreza o vulnerabilidad (CONEVAL, 2016).

Si en los informes del DIF y en la visión de esta instancia que consta en la página oficial se señala un claro avance en su objetivo de "mitigar la situación de marginación y exclusión de los sectores más vulnerables y a fortalecer a la familia como factor fundamental del desarrollo y bienestar humano", ${ }^{7}$ surge la necesidad de analizar esa eficacia institucional a la que se ha hecho referencia a partir de casos concretos. Se retomará un ejemplo para la reflexión.

\section{La intervención de la DAIF hacia los vulnerables}

La DAIF interviene a solicitud de las autoridades municipales, quienes en todo momento fungen como enlace entre la población y la institución:

[...] nosotros intervenimos [...] vemos toda la parte de prevenir los riesgos psicosociales. Es decir, tenemos varias líneas de acción para poder entrar al municipio. En primer lugar, que el municipio lo solicite. El municipio nos manda un oficio de solicitud, inclusive damos pláticas a servidores públicos, servidores públicos tanto del municipio como los policías, por ejemplo. ${ }^{8}$

Por la manera como se establece el contacto entre el municipio y la institución, la intervención de la DAIF se realiza sin que su personal tenga conocimiento a priori del contexto situado en el cual trabajará. Es decir, los talleres o pláticas para prevenir riesgos en la población vulnerable se diseñan a partir de lo que sus empleados consideran adecuado de acuerdo con la problemática reportada por el municipio, y desde lo que abstraen de sus propias reflexiones como posible solución a la situación de riesgo. Además, el contenido de los talleres se replica indistintamente en todos municipios sin una reflexión sobre las diferencias históricas, económicas, sociales y de vulnerabilidad entre las distintas regiones del estado. En este sentido, una de las trabajadoras sociales definió su labor de la siguiente manera: "mi trabajo es dar talleres a los pueblos, talleres de lo que pidan".

Para ahondar en una problemática concreta tratada en la DAIF, se retomará a manera de ejemplo el embarazo adolescente, tema importante que urge atender, tal y como se señala en la Estrategia Nacional para la Prevención del Embarazo en Adolescentes (ENAPEA). De acuerdo con el informe ejecutivo 2015 de la ENAPEA (2016), 466900 niñas y adolescentes entre los 10 y 19 años se convirtieron en madres durante el año 2013 en el país. En la página oficial del gobierno de la República Mexicana, y con relación al embarazo adolescente, se señala que México ocupa el primer lugar entre los países de la Organización para la Cooperación y el Desarrollo Económico (OCDE) en relación con esta problemática: su tasa de fecundidad es de 77 nacimientos por cada mil adolescentes de 15 a 19 años de edad. Investigadores como Claudio Stern han analizado ese fenómeno desde la vulnerabilidad social en diferentes contextos situados de México, y han llegado a conclusiones que explican los embarazos en adolescentes a partir de una combinación de diversas variables entre las que destaca el componente sociocultural (Stern, 2003 y 2004).

En el caso de estudio, si bien la DAIF identifica acertadamente que los embarazos en adolescentes constituyen una condición de riesgo, parte de ubicar a las mujeres como piezas importantes en el problema, por lo que se planifican capacitaciones prioritariamente para ellas; además, como reconoció una de las promotoras de la institución, "los hombres no participan". Las pláticas o talleres son intervenciones breves que se centran en la promoción y utilización de métodos anticonceptivos y en las circunstancias de pobreza en las cuales nacerían los hijos. Los facilitadores realizan las presentaciones sin conocer el contexto local ni la complejidad de un problema de alcance nacional como lo es el embarazo adolescente, por lo que sus intervenciones presentan un tono superficial.

Otro punto que merece atención es la falta de seguimiento que desde la institución se hace a los grupos identificados en riesgo. Ciertamente, aunque ésta es una labor compleja e incluso cercana a lo imposible, 
una vía plausible consiste en articular esfuerzos entre instituciones, en vez de duplicarlos (Flores y Rubio, 2017). En el caso del embarazo en adolescentes, si ya se conoce que existe la problemática, es importante cuestionar la pertinencia de seguir impartiendo talleres año tras año, los cuales tienen bajo impacto en las subjetividades como se deduce del análisis de las cifras oficiales (UNICEF, 2015). ${ }^{10}$ Se parte de que el embarazo adolescente es una problemática cuyo origen se finca en representaciones hegemónicas que sustentan y estructuran las prácticas sexuales en el contexto, por lo que éstas difícilmente pueden deconstruirse, o siquiera cuestionarse, mediante pláticas sobre métodos anticonceptivos. Ante el desconocimiento de las implicaciones de la problemática y de los contextos situados, estos talleres y pláticas sobre salud reproductiva difícilmente podrán impactar en las poblaciones intervenidas. El embarazo adolescente es una práctica que se sustenta en diversas situaciones sociales, a manera de "vulnerabilidades acumuladas", tal como reportaron Flores y Mora (2010) para otro contexto de investigación. La pobreza, la marginación, el racismo y la inequidad son algunas de las causas que se encuentran en la base de la problemática, por lo cual no bastan los talleres para transformar una conciencia construida desde la exclusión y el no reconocimiento.

Pareciera que, a partir del ejemplo del embarazo en adolescentes, no se concibe una estrategia para impulsar los activos desde la capacidad de afrontar la vulnerabilidad, sino que las acciones se concentran en paliativos, como son los talleres. Villa señala en este sentido que:

La idea del fortalecimiento de los activos presenta la posibilidad de propiciar acciones proactivas y preventivas, que trasciendan los alcances de las intervenciones de tipo paliativo, aunque sin excluir la utilización de éstas para hacer frente a los efectos adversos de las transformaciones económicas, sociales y culturales de la región (Villa, 2001: 2)

Se considera que la intervención sin conocimiento del contexto social y de la problemática puede estar condenada al fracaso. ¿Cuál es el sentido de dar pláticas o talleres si se desconoce la realidad y el modo de vida de la población a quien van dirigidos? Es justamente en este punto donde el tema de las representaciones sociales es importante porque implica ese necesario conocimiento de la realidad, de ese saber de sentido común que toda persona posee a partir de sus experiencias de vida.

\section{Conclusiones}

Un estudio de vulnerabilidad amerita diversas precisiones entre las que destacan la variable explicativa de la situación de riesgo a enfatizar, las condiciones estructurales implícitas en ese mismo entorno, y el contexto situado que la configura y le imprime un sesgo particular. También es fundamental retomar los activos sociales, esas "vulnerabilidades recursivas" (Flores, 2015) que están presentes en la cotidianidad de las experiencias de hombres y mujeres en sus contextos, o que se construyen ante la adversidad. Desde la apreciación de las autoras de este texto, la vulnerabilidad corresponde a procesos estructurales que se explican a partir de la convergencia de múltiples variables configuradas en los contextos situados y en la propia historia individual o colectiva. Es un concepto dinámico y relativo que reivindica a los mismos grupos e individuos al reconocerlos capaces de identificar y transformar sus condiciones de vida.

En este artículo se ha mostrado que el concepto "vulnerabilidad" es empleado de una manera particular en las instituciones que trabajan en política social en Yucatán, específicamente en la DAIF. Su uso suele equipararse a precariedad material o cognitiva, por lo que se desvirtúa el sentido real del concepto y, con ello, se reducen las posibilidades de incidir en las condiciones de vida de la población en riesgo multifactorial. En este sentido, la institución crea las condiciones de la experiencia personal o comunitaria de situaciones de riesgo o vulnerabilidad, como el ejemplo analizado del embarazo adolescente. Visibilizar este condicionamiento es un aporte de la etnografía institucional. 
Sin embargo, existen posibilidades de transformar las condiciones de vulnerabilidad desde los contextos situados, reconociendo a los actores como sujetos sociales y potenciando sus capacidades de tal manera que desde las bases se gesten agendas de trabajo con sustento. Por ello, se proponen a continuación algunas líneas de acción como herramientas para consolidar estrategias de intervención eficaces. En otro espacio (Flores y Rubio, 2017) bosquejamos algunas de estas líneas, y agregamos a éstas la importancia de la reflexión que origina y explica las condiciones en las que se desarrolla la población vulnerable. Esto equivaldría a analizar las mismas políticas públicas y sus programas correspondientes en escenarios específicos, aquellos que no son visibles en los datos cuantitativos que permean el discurso oficial de programas y direcciones como los documentados en este artículo; y desde luego, a ponderar el efecto de las intervenciones gubernamentales en los contextos situados.

Otra línea consiste en señalar la necesidad de que el personal a cargo de operar los programas conozca las temáticas de intervención. En el caso del embarazo en adolescentes, se observó un tratamiento superficial del tema sin dimensionar sus implicaciones, sus orígenes ni las representaciones que lo sustentan. Por ello, el tratamiento del problema no tuvo mayor impacto. En este sentido, sería conveniente la incorporación de expertos en temas de riesgo como el embarazo adolescente, VIH-Sida, violencia de género o acoso escolar, para capacitar a los promotores y al personal que tiene a su cargo trabajar con los grupos vulnerables. Por último, es importante ajustar los talleres a las necesidades de los grupos de intervención y a sus contextos situados. Los referentes históricos y socioculturales que dan sentido y explican los escenarios sociales también son elementos clave para organizar los contenidos temáticos de cada intervención. Un metaanálisis de problemáticas como las mencionadas implica develar el escenario social que las sustenta y configura; la intervención no puede prescindir de dicho metaanálisis.

\section{Notas}

${ }^{1}$ Los informes de actividades de la presidenta del DIF se encuentran disponibles en audio desde el segundo año de actividades (2014). En 2016 la presentación fue transmitida vía redes sociales

${ }^{2}$ A lo largo de su experiencia en la política local, la directora de la DAIF ha estado al frente de diversos programas y direcciones en desarrollo social de la entidad.

${ }^{3}$ Entrevista a empleada de la DAIF, 69 años, 2017.

${ }^{4}$ Entrevista a empleado de la DAIF, 30 años, 2017.

${ }^{5}$ Entrevista a empleado de la DAIF, 41 años, 2017.

6 En: http://www.dif.yucatan.gob.mx/quienes_somos. html (consultado el 19 de enero de 2017).

7 En: http://www.dif.yucatan.gob.mx/quienes_somos. html (consultado el 19 de enero de 2017).

${ }^{8}$ Entrevista a empleado de la DAIF, 41 años, 2017.

${ }^{9}$ Entrevista a empleada de la DAIF, 36 años, 2017.

${ }^{10}$ UNICEF México reporta en su informe 2015 que el 9.55\% del total de las adolescentes de entre 15 y 17 años que viven en el país han estado embarazadas en algún momento de sus vidas, y que los estados con mayor porcentaje de embarazos en adolescentes son Coahuila, Hidalgo y Michoacán.

\section{Referencias}

Busso, Gustavo (2001). "Vulnerabilidad social: nociones e implicancias de políticas para Latinoamérica a inicios del siglo XXI". Ponencia presentada en el Seminario Internacional Las Diferentes Expresiones de la Vulnerabilidad Social en América Latina y el Caribe. Disponible en: http://www.cepal.org/ publicaciones/xml/3/8283/GBusso.pdf (consultado el 22 de junio de 2017).

CONAPO (Consejo Nacional de Población) (2011). Índice de marginación por entidad federativa y municipio 2010. México: CONAPO.

CONAPO (Consejo Nacional de Población) (2016) Índice de marginación por entidad federativa y municipio 2015. México: CONAPO.

CONEVAL (Consejo Nacional de Evaluación de la Política de Desarrollo Social) (2016). Informe de 
evaluación de la política de desarrollo social 2016. México: CONEVAL

Estrada, Adriana (2014). "Forma de pensar de mujeres indígenas migrantes frente a la discriminación social". En Úrsula Oswald et al., Vulnerabilidad social y género entre migrantes ambientales. México: Centro Regional de Investigaciones Multidisciplinarias de la Universidad Nacional Autónoma de México, pp. 293-316.

ENAPEA (Estrategia Nacional para la Prevención del Embarazo en Adolescentes) (2016). Informe 2016. Disponible en: https://bit.ly/2Iu2UId (consultado el 20 junio 2017).

Flores, Fátima (2015). Experiencia vivida, género y VIH. Sus representaciones sociales. México: Universidad Nacional Autónoma de México.

Flores, Fátima y Amada Rubio (2017). “Las instituciones públicas en el Estado de Yucatán: Retos y Desafíos en el bienestar social". En Gaceta Legislativa del Estado de Yucatán, l, enero-marzo. Disponible en: http://www. congresoyucatan.gob.mx/vistas/gaceta/2017/1/ index.html (consultado el 22 de junio de 2017).

Flores, Fátima y Jazmín Mora (2010). "Pobres, enfermas y locas, una historia de vulnerabilidades acumuladas". En María Montero (ed.), La ecología social de la pobreza. Impactos psicosociales, desafíos multidisciplinarios. México: Universidad Nacional Autónoma de México, pp. 79-108.

Gobierno del Estado de Yucatán(1986). Ley sobreel Sistema Estatal de Asistencia Social de Yucatán. Decreto núm. 353. Disponible en: http:/www.congresoyucatan.gob. mx/detalle_ley.php?idley=117 (consultado el 20 de junio de 2017).

Gobierno del Estado de Yucatán (2013). Plan estatal de desarrollo 2012-2018 Yucatán. Disponible en: http://www.yucatan.gob.mx/gobierno/ped.php (consultado el 19 de enero de 2017).

Gobierno del Estado de Yucatán (2017). Catálogo de programas de bienes y servicios Yucatán 2017. Disponible en:http://www.yucatan.gob.mx/ciudadano/ programas.php (consultado el 11 de mayo de 2017).

Hevia, Felipe (2009). "De Progresa a Oportunidades: efectos y límites de la corriente cívica en el gobierno de Vicente Fox". En Sociológica, 70: 43-81.
INEGI (Instituto Nacional de Estadística y Geografía) (2016). Indicador trimestral de la actividad económica estatal. México: INEGI.

ONU (Organización de las Naciones Unidas) (2014). México: desafíos para un desarrollo incluyente. México: ONU.

Pineda, Siboney, Ivonne Vizcarra y Bruno Lutz (2006). "Gobernabilidad y pobreza: proyectos productivos para mujeres indígenas mazahuas del Estado de México". En Indiana, 23: 283-307.

Pizarro, Roberto (2001). La vulnerabilidad social y sus desafíos: una mirada desde América Latina. Santiago de Chile: Naciones Unidas.

Rubio, Amada (2018). "Experiencia vivida por mujeres mayas en proyectos productivos: retos y oportunidades para la agenda de desarrollo de los pueblos indígenas". En Estudios de Cultura Maya, 51: 229-256.

Rubio, Amada y María Teresa Castillo (2014). "Mujeres mayas en Yucatán: experiencia participativa en una organización productiva". En Convergencia. Revista de Ciencias Sociales, 64: 39-63.

Sáez Carreras, Juan, Sacramento Pinazo Hernandis y Mariano Sánchez Martínez (2008). “La construcción de los conceptos y su uso en las políticas sociales orientadas a la vejez: la noción de exclusión y vulnerabilidad en el envejecimiento". En Revista del Ministerio de Trabajo e Inmigración, 75: 75-94.

Smith, Dorothy (2005). Institutional Ethnography. A Sociology for People. Toronto: Altamira Press.

Soares, Denise, María Teresa Munguía, Gemma Millán, Jorge Villarreal, Hilda Salazar y Germán Méndez (2014). Vulnerabilidad y adaptación en Yucatán. Un acercamiento desde lo local y con enfoque de género. México: Instituto Mexicano de Tecnología del Agua.

Stern, Claudio (2003). "Significado e implicaciones del embarazo adolescente en distintos contextos socioculturales de México: reseña de un proyecto en proceso". En Estudios Sociológicos, 3: 725-745.

Stern, Claudio (2004). "Vulnerabilidad social y embarazo adolescente en México". En Papeles de Población, 39: 129-158. 
UNICEF (2015). Informe anual 2015. México: UNICEF. Villa, Miguel (2001). Vulnerabilidad social: notas preliminares. Santiago de Chile: Naciones Unidas.

Villagómez, Gina y María Sánchez (2013). “Tercera edad y pobreza. Algunas aristas de la desigualdad social en Campeche". En Gina Villagómez y Ligia Vera (coords.), Vejez. Una perspectiva sociocultural. México: Universidad Autónoma de Yucatán, Universidad Autónoma de Campeche, pp. 123-150.

Villagómez, Gina (2014). "Mujeres mayas: envejecimiento, pobreza y vulnerabilidad". En Península, 2: 75-98.
Vizcarra, Ivonne (2002). "La institucionalización de la equidad de género en el Estado de México y la economía política feminista”. En Convergencia. Revista de Ciencias Sociales, 30: 79-95.

Vizcarra, Ivonne y Fernando Farfán (2012). "El programa 'vete sano y regresa sano': lecciones aprendidas de una etnografía institucional en el Estado de México". En Ruris. Revista do Centro de Estudios Rurais, 2: 123-154.

Zapata, Emma y Blanca Suárez (2007). "Las artesanas, sus quehaceres en la organización y en el trabajo". En Ra Ximhai. Revista de Sociedad, Cultura y Desarrollo Sustentable, 3: 591-620. 